\title{
Nucleosynthesis of Light-Element Isotopes in Evolved Stars Experiencing Extended Mixing
}

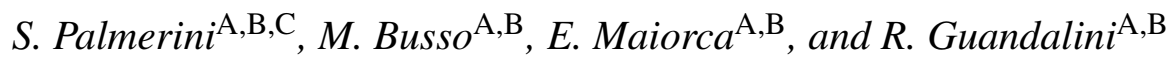 \\ A Dipartimento di Fisica, Università degli Studi di Perugia, via Pascoli, 06123 Perugia, Italy \\ ${ }^{B}$ INFN Sezione di Perugia, via Pascoli, 06123 Perugia, Italy \\ ${ }^{\mathrm{C}}$ Corresponding author. Email: palmerini@ fisica.unipg.it
}

Received 2008 November 18, accepted 2009 May 26

\begin{abstract}
We present computations of nucleosynthesis in red giants and Asymptotic Giant Branch (AGB) stars of Population I experiencing extended mixing. The assumed physical cause for mass transport is the buoyancy of magnetized structures, according to recent suggestions. The peculiar property of such a mechanism is to allow for both fast and slow mixing phenomena, as required for reproducing the spread in $\mathrm{Li}$ abundances displayed by red giants and as discussed in an accompanying paper. We explore here the effects of this kind of mass transport on $\mathrm{CNO}$ and intermediate-mass nuclei and compare the results with the available evidence from evolved red giants and from the isotopic composition of presolar grains of AGB origin. It is found that a good general accord exists between predictions and measurements; in this framework we also show which type of observational data best constrains the various parameters. We conclude that magnetic buoyancy, allowing for mixing at rather different speeds, can be an interesting scenario to explore for explaining together the abundances of $\mathrm{CNO}$ nuclei and of Li.
\end{abstract}

Keywords: nuclear reactions, nucleosynthesis, abundances — stars: AGB — stars: chemically peculiar — stars: evolution

\section{Introduction}

The atmospheric composition of evolved red giants is known to result from the interplay of nuclear and dynamical processes, the latter transporting material from the burning shells to the envelope (Busso, Gallino \& Wasserburg 1999); these phenomena are also recognized to depend strongly on the stellar metallicity (Straniero et al. 2006). The best known outcome of the above interplay is the appearance in the photosphere, during the Asymptotic Giant Branch (AGB), of heavy nuclei generated by slow neutron captures in the so-called $s$ process (Käppeler et al. 1989). The main neutron source for the neutrons was identified in $(\alpha, \mathrm{n})$ reactions on ${ }^{13} \mathrm{C}$, occurring in the radiative He-rich layers (Gallino et al. 1998).

Other chemical peculiarities accompany the advanced evolution of low mass stars (LMS). They are only partly observed by stellar spectroscopy (Harris, Lambert \& Smith 1988; Smith \& Lambert 1990; Wallerstein \& Morell 1994), important constraints on them deriving also from high accuracy measurements of isotopic shifts in presolar grains of AGB origin (Nittler et al. 1997; Choi et al. 1998; Amari, Zinner \& Lewis 2000; Zinner et al. 2007). This includes, for example, the ratios ${ }^{12} \mathrm{C} /{ }^{13} \mathrm{C},{ }^{17} \mathrm{O} /{ }^{16} \mathrm{O}$, ${ }^{18} \mathrm{O} /{ }^{16} \mathrm{O},{ }^{26} \mathrm{Al} /{ }^{27} \mathrm{Al}$.

After the works by Gilroy (1989) and Gilroy \& Brown (1991), it became clear that canonical stellar models (including only purely convective mixing) are unable to account for the whole set of changes affecting the isotopic mix of light and intermediate-mass nuclei, which begin to appear during the first ascent of the Red Giant Branch (RGB). Some additional transport mechanism is required (Boothroyd, Sackmann \& Wasserburg 1995; Charbonnel \& Do Nascimento 1998; Charbonnel \& Balachandran 2000; Nollett, Busso \& Wasserburg 2003), in which part of the envelope material is carried down to regions of sufficiently high temperature to undergo proton capture.

Such mixing phenomena were studied in several works (Charbonnel 1994; Wasserburg et al. 1995; Sackmann \& Boothroyd 1999) and named in various ways: extended mixing, deep mixing, extra-mixing and cool bottom processes (or CBP). They were often attributed to rotationally induced effects (Charbonnel 1994; Charbonnel \& Do Nascimiento 1998), however it was subsequently demonstrated that the reaction of the stellar structure to a centrifugal distortion would be too fast to allow for extended transport of matter on long time-scales (Palacios et al. 2006).

More recently, it was noticed by Eggleton, Dearborn \& Lattanzio (2006) that important mixing processes can be induced in stars by the molecular weight inversion generated in the reaction ${ }^{3} \mathrm{He}+{ }^{3} \mathrm{He} \rightarrow{ }^{4} \mathrm{He}+2 p$, implementing a form of thermohaline diffusion (Charbonnel \& Zahn 2007).

Alternative (or complementary) mechanisms exist: it was in particular underlined that the presence of a magnetic dynamo would permit the buoyancy of magnetized 
structures created near the H-burning shell, thus inducing a form of matter circulation in RGB and AGB stages (Busso et al. 2007; Nordhaus et al. 2008; Denissenkov, Pinsonneault \& MacGregor 2009). Recently, similar buoyancy processes for the Sun have been shown to reproduce a number of details of the matter upflows and downflows and other known features of the solar dynamo (Li, Sofia \& Belvedere 2005).

One has also to notice that extra-mixing during the AGB stage has to account for the presence, in presolar grains of circumstellar origin, of nuclei (like ${ }^{26} \mathrm{Al}$ ) that are synthesized well below the layers where ${ }^{3} \mathrm{He}$ burns, so that in late evolutionary stages thermohaline mixing alone might not suffice (Palmerini \& Busso 2008). This process, with its typical low velocity, should be dominant in the long-lasting main sequence phases, where it might account for the element changes occurring in the Sun and in similar stars (Melendez \& Ramirez 2007). On the other hand, the scheme of magnetic buoyancy devised by Busso et al. (2007) can be faster, hence more suitable for the postmain-sequence evolutionary stages, where in fact several classes of magnetically active stars do exist (Andrews et al. 1988).

In a paper presented in this volume (Guandalini et al. 2009) we analyzed the advantages that magnetic buoyancy might offer for explaining the complex phenomenology of Li production and destruction in red giants. Hereinafter we shall refer to that work as to 'Paper I'. In the present work we want to derive the effects that the same models would induce on $\mathrm{CNO}$ and other intermediate-mass nuclei, in order to verify the compatibility of that idea with a wider set of observational constraints. In Section 2 we discuss the models adopted; in Sections 3 and 4 we present some comparisons with abundance observations in stars and presolar grains, respectively. Finally, in Section 5 preliminary conclusions are drawn.

\section{Buoyancy and Mixing}

As mentioned, several models of the coupled occurrence of nucleosynthesis and extended mixing were presented in the past. In particular, Nollett, Busso \& Wasserburg (2003) proposed a scheme based on a conveyor-belt-like circulation for transporting matter between the base of the convective envelope and the region immediately above the H-burning shell, also showing that this approach is equivalent to a diffusion treatment. The work contained two main free parameters, namely the mixing rate $\dot{M}$ and the maximum temperature experienced by the circulating material, $T_{\mathrm{P}}$; its results suggested that the observed isotopic shifts in presolar grains require $\dot{M}$ values in the broad range $10^{-7}$ to $10^{-5} \mathrm{M}_{\odot} \mathrm{yr}^{-1}$ and $T_{\mathrm{P}}$ values such that $\left(\log T_{\mathrm{P}}-\log T_{\mathrm{H}}\right) \geq 0.1$, where $T_{\mathrm{H}}$ is the temperature of the H-burning shell, defined as the one at which the maximum energy is generated.

In a subsequent work by Busso et al. (2007) it was suggested that the driving force for mixing might be provided by the buoyancy of magnetic flux tubes formed near the H-burning shell. Those authors derived, for the speed of this buoyancy, the relation:

$$
v(r)=\frac{1}{2}\left(\frac{\rho(r)}{\rho_{0}}\right)^{3 / 4}\left(\frac{r}{r_{0}}\right)^{-1 / 4}\left(\frac{g_{0} a_{0}}{C_{\mathrm{D}}}\right)^{1 / 2} \frac{B_{0}}{\sqrt{P(r)}}
$$

(see Busso et al. 2007, for details). In the above equation $B_{0}$ is the magnetic field somewhere near the H-burning shell (where $T=T_{\mathrm{P}}$ ), $a_{0}$ is the initial radius of flux tubes in the same position, $C_{\mathrm{D}}$ is the aerodynamic drag coefficient, and the other quantities (pressure, temperature and density) are given by the stellar structure, as computed with the FRANEC code. Since heat exchanges between magnetized and non-magnetized matter are not considered in this approach, the buoyancy is quite fast; it starts with an upward motion, which also induces (by mass conservation) a downflow. Fast matter transport driven by magnetic buoyancy is indeed observed in the Sun (Briand \& Solanki 1998).

In the calculations of the present paper we shall assume that the above prescriptions (included in what we shall define as 'Model A') be valid for the RGB phases immediately after the first dredge-up. These stages witness the repeated crossing, by the point representing a star, of the same zone in the HR diagram, so that these points pile up and the luminosity function (LF) displays a 'bump'. Stars at the LF-bump have just experienced the advancement of the H-burning shell through the composition discontinuity left behind by the first dredge-up, so that the radiative region below the convective envelope has no gradient in molecular weight, and any mixing phenomenon can occur easily. In these stages, as early noticed by Charbonnel \& Balachandran (2000), Li is enhanced, most probably by a mixing mechanism mimicking the so-called CameronFowler process, i.e. a transport of ${ }^{7} \mathrm{Be}$ to the surface at a speed fast enough to prevent it from experiencing proton or electron captures along the path (Cameron \& Fowler 1971). In Paper I we showed that a mixing scheme like that envisaged by Busso et al. (2007) can in fact account for the observed Li production. With the application of Model A we want here to infer the corresponding effects on $\mathrm{CNO}$ and intermediate-mass nuclei.

It is however well known from solar observations that the dynamical behaviour of photospheric structures (including those generated by magnetic dynamos) is complex in nature and displays motions at very different

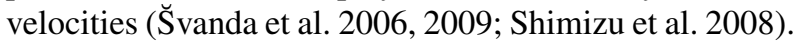
No observational information on such processes in red giants, on their speed and on the fractional area they might cover are within our reach. It was however underlined recently (Denissenkov et al. 2009) that the buoyancy of large structures in red giants should occur at a velocity lower than estimated by Busso et al. (2007), because heat exchanges with the environment grow with the surface of the rising 'bubbles'. Slow transport should favour Li destruction. Guided by this consideration and by Li observations in RGB and AGB stars, in Paper I we assumed that, after the first fast phase, the buoyancy slows down to an extra-mixing process similar to those previously explored 
by Wasserburg et al. (1995) and by Nollett, Busso \& Wasserburg (2003), so that the Li abundances decrease. In order to verify the effects on $\mathrm{CNO}$ isotopes and other intermediate nuclei of those assumptions we, too, explore this possibility: it will be referred to as 'Model B'.

Concerning the actual speed of the mixing processes, in Model A we assume for it a form like in Equation 1, but with a suitable scaling. The equation was derived by Busso et al. (2007), from considering that an adequate recycling of the RGB envelope is obtained by releasing about $\dot{N} \simeq 3 \times 10^{-8}$ flux tubes per second. If we want now to refer not to entire flux tubes, but to small magnetized structures capable of moving at a fast rate (e.g. parcels of magnetized material less massive than, say, one tenth of a flux tube or so), then we need a higher release rate (by a factor of ten in this case). We must then verify that the rising velocity, although high, remains realistic, in particular not larger than the Alfvén speed. Let now $\hat{v}$ be the average buoyancy velocity over the radiative layers, and let the distance covered by the buoyancy be $\Delta r$; from the model by Busso et al. (2007) for an RGB star of $1.5 \mathrm{M}_{\odot}$, using their Equation 1:

$$
\dot{N}=\frac{\hat{v}}{\Delta r}
$$

and assuming, as done in Paper $\mathrm{I}, \log T_{\mathrm{H}}-\log T_{\mathrm{P}}=0.3$, one gets $\Delta r=5 \times 10^{10} \mathrm{~cm}$ and $\hat{v}=100 \mathrm{~m} \mathrm{~s}^{-1}$. For the average density of the radiative layers below the envelope in RGB phases, this value is lower than the Alfvén velocity $\left(B /(4 \pi \bar{\rho})^{1 / 2}\right)$ if $B$ is larger than about $10^{4} \mathrm{G}$. This condition is satisfied even in the solar tachocline and should therefore be easily met. We conclude that Model A is not physically unreasonable.

For the second case (Model B) we assume, as made in Nollett, Busso \& Wasserburg (2003), that the circulation is slow, both along the ascending and the descending path; for the sake of simplicity we assume that the two flows occupy the same fractional surface $f=0.5$ (at any distance from the stellar centre). The velocity of the flow is in this case set by:

$$
v(r)=\frac{\dot{M}}{4 \pi r^{2} \rho f}
$$

For the range of $\dot{M}$ values discussed in Paper I we obtain $v$ values (in $\mathrm{cm} \mathrm{s}^{-1}$ ) ranging from a few tenths to a few. The choices of $T_{\mathrm{P}}$ are adopted from Paper I, as they guarantee that the observed Li abundances in RGB and AGB stars be reproduced. We then solve the set of equations describing the abundance changes in the mixed material, due to the nucleosynthesis and displacement processes. They can be expressed through the total derivatives:

$$
\frac{\mathrm{d} N_{i}}{\mathrm{~d} t}=\frac{\partial N_{i}}{\partial t}+\frac{\partial N_{i}}{\partial M} \frac{\partial M}{\partial R} \frac{\partial R}{\partial t}
$$

where the partial time derivative is due to nucleosynthesis and the second term is due to mixing. For the nuclear parameters we adopted the NACRE compilation (Angulo et al. 1999).

We study here AGB models of initially $1.5 \mathrm{M}_{\odot}$, at two different metallicities $\left(Z=Z_{\odot} / 2\right.$, and $\left.Z_{\odot} / 3\right)$. We also include the case of a $2 \mathrm{M}_{\odot}$ star of solar metallicity. Of the above models, the first one never becomes $\mathrm{C}$-rich, and its predicted composition can be compared with those of O-rich AGB stars and of presolar oxide grains (Choi et al. 1998). The others do become carbon stars; their predictions can be compared with the abundance observations in $\mathrm{C}(\mathrm{N})$ giants and in presolar $\mathrm{SiC}$ grains (Zinner et al. 2007).

The effects of extra-mixing in RGB and AGB stars are studied though a post-processing code that also reproduces the effects of the third dredge up (TDU), occurring on the AGB after advanced thermal pulses of the Heburning shell. The TDU contribution is important for several nuclei, in particular for ${ }^{12} \mathrm{C},{ }^{22} \mathrm{Ne},{ }^{26} \mathrm{Al}$, as well as for neutron-capture nuclei produced in the He-intershell zone (which are, however, not considered here due to space limitations).

It is well known that the efficiency of TDU decreases with increasing metallicity (Busso et al. 2001). In our analysis the timing and mixed mass of each dredge-up episode are calculated from the physical structure of the original stellar models, using the analytical expressions suggested in Straniero et al. (2003). The composition of the dredgedup material, in terms of the light nuclei we study here, is recomputed on the basis of the models presented by Wasserburg et al. (2006).

The duration of the thermally-pulsing AGB stage is controlled by mass loss; its efficiency is unfortunately an unknown parameter. For illustration purposes we adopt here the simple parametrization by Reimers (1975) fixing the free parameter $\eta$ to unity. With this choice the final compositions of the models considered are characterized by $\mathrm{C} / \mathrm{O}$ ratios below 1 for the $1.5-\mathrm{M}_{\odot}$ model with $Z=Z_{\odot} / 2$; of up to 2 for the $1.5-\mathrm{M}_{\odot}, Z=Z_{\odot} / 3$ case; and of up to 1.5 for the $2.0-\mathrm{M}_{\odot}, Z=Z_{\odot}$ model. The actual $\mathrm{C} / \mathrm{O}$ value reached at the end of each calculation depends on the effectiveness assumed for CBP, in particular on $\dot{M}$ (lower $\dot{M}$ values allowing higher ${ }^{12} \mathrm{C}$ abundances to be reached).

\section{Results and Discussion}

During the evolution, the mixing events mentioned in the previous Section cause the envelope composition to change gradually in time. Both elemental and isotopic abundances are affected and the isotopic mix of each element is followed in detail as the time proceeds. Our computations produce sequences like those illustrated in Figures 1 to 3 .

In particular, Figure 1 illustrates the effects on $\mathrm{CNO}$ isotopic ratios of applying Model A, with circulation rates $\dot{M}$ in the range $(0.05-1) \times 10^{-6} \mathrm{M}_{\odot} \mathrm{yr}^{-1}$, until the bolometric magnitude reaches about zero, exactly as done in Paper I for reproducing the $\mathrm{Li}$ abundances at the L-bump. In this way we can infer the isotopic shifts for intermediate mass elements expected from the same mixing 


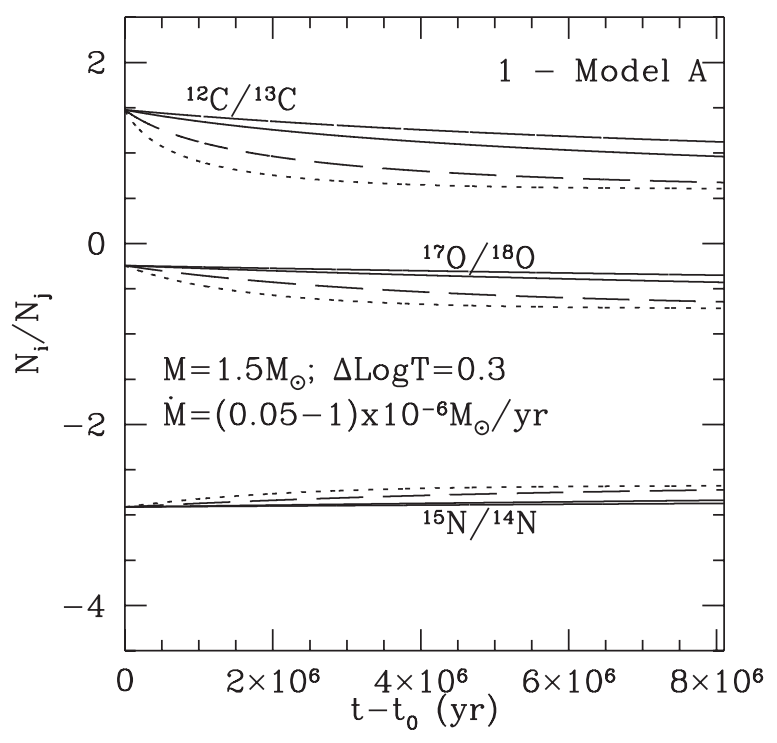

Figure 1 The temporal evolution of $\mathrm{CNO}$ isotopic ratios in the envelope of an RGB model with $M=1.5 \mathrm{M}_{\odot}$ and half solar metallicity. The ratios derive from the operation of CBP, following the prescription of Model A (see text for explanations). Different lines refer to different choices for $\dot{M}$. Dots, short dashes, continuous line and long dashes correspond to $\dot{M}=1,0.6,0.4$ and 0.1 (in units of $\left.10^{-6} \mathrm{M}_{\odot} \mathrm{yr}^{-1}\right)$, respectively.

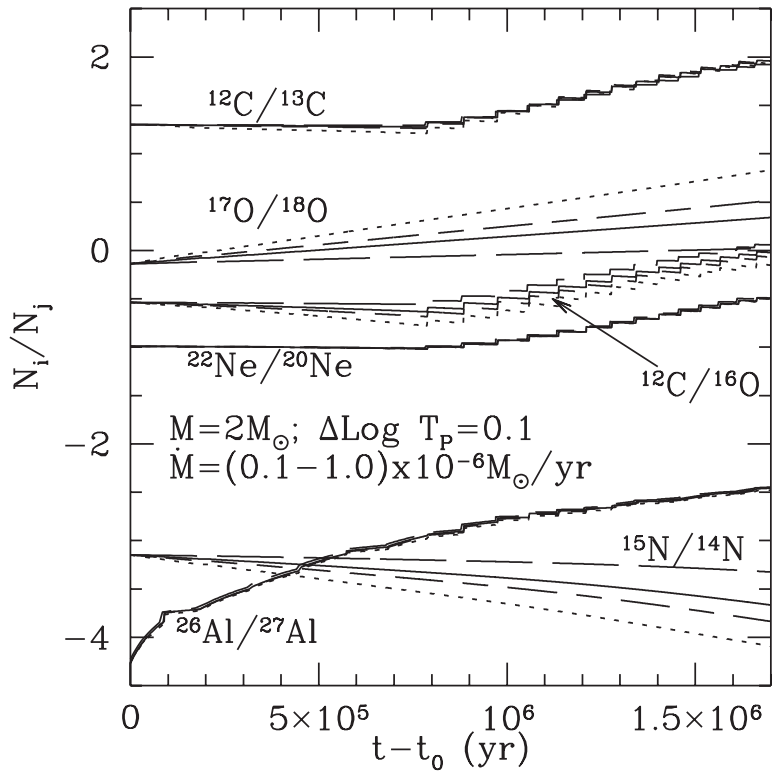

Figure 2 The temporal evolution of isotopic ratios for $\mathrm{CNO}$ and $\mathrm{Al}$ in the envelope of an AGB model with $M=2 \mathrm{M}_{\odot}$ and solar metallicity. The ratios derive from the combined operation of CBP and TDU. The plot shows the effects of changing the circulation rate $\dot{M}$ within a limited interval. Different lines refer to different choices for $\dot{M}$. Dots, short dashes, continuous line and long dashes correspond to $\dot{M}=1,0.6,0.4$ and 0.1 , (in units of $10^{-6} \mathrm{M}_{\odot} \mathrm{yr}^{-1}$ ) respectively.

scheme already shown to be suitable for explaining the $\mathrm{Li}$ abundances. As shown by the Figure, a transport at high speed, down to regions of moderately high temperature ( $\Delta \log T=0.3$ ), as implied by Model $\mathrm{A}$, has detectable effects on few isotopes, and mainly on the ${ }^{12} \mathrm{C} /{ }^{13} \mathrm{C}$ ratio. All the cases shown in Figure 1 do produce $\mathrm{Li}$ at the level required by observations, if the mixing is performed

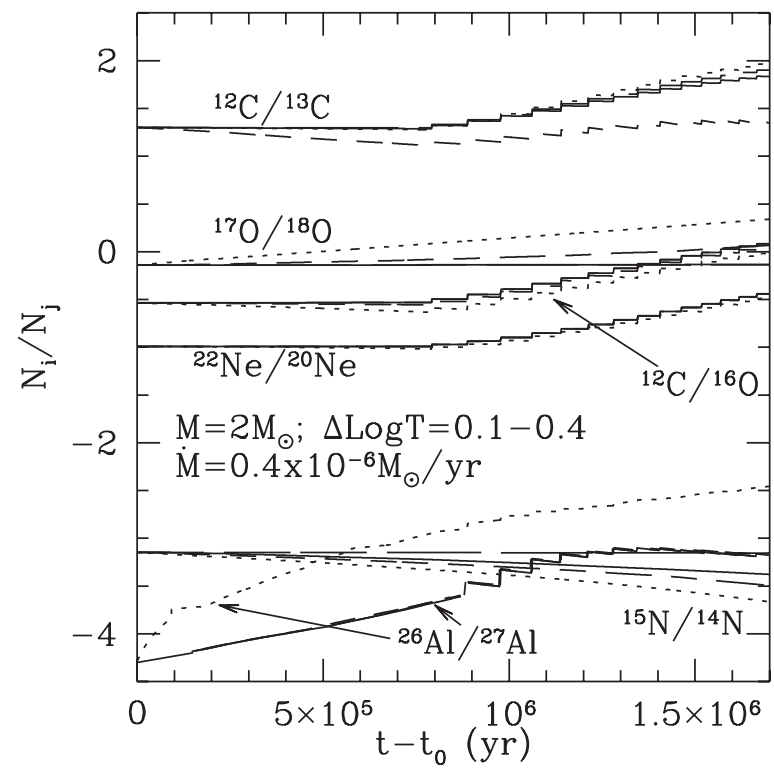

Figure 3 Same as Figure 2, but showing the effects induced by changing the $\operatorname{logarithmic}$ separation $\Delta \log T$, between the H-shell temperature $T_{\mathrm{H}}$ and the maximum temperature achieved by the circulating material $T_{\mathrm{P}}$. Different lines refer to different choices for $\Delta \log T$. Dots, short dashes, continuous line and long dashes correspond to $\Delta \log T=0.1,0.2,0.3$ and 0.4 , respectively.

through the intermittent release of magnetized bubbles traveling at high velocity. As the carbon isotopic mix is modified differently by mixing with different $\dot{M}$ rates, we expect that stars at the L-bump, showing Li in their spectra with abundances up to $\log \epsilon(\mathrm{Li})=2-2.5$ (see Paper I) display a spread in carbon isotopic ratios directly related to the $\dot{M}$ value. This effect is weaker for $\mathrm{N}$ and $\mathrm{O}$ isotopes. All the curves of Figure 1 have, in their terminal part, a slope close to zero. This depends on the fact that the mixing has been applied only down to regions where $\Delta \log T=0.3$, rather far from the H-burning shell, where the nucleosynthesis effects are moderate. In these zones the ${ }^{12} \mathrm{C} /{ }^{13} \mathrm{C}$ ratio is not at its typical $\mathrm{H}$-burning equilibrium value (near $3.5) ;{ }^{12} \mathrm{C}$ is only marginally affected by $p$-captures, producing some ${ }^{13} \mathrm{C}$. Since these effects are rather small, during the $8 \times 10^{6} \mathrm{yr}$ spent in mixing according to Model A, the envelope abundances have time to reach almost their asymptotic values. The subsequent switch to a slower transport (Model B), while destroying Li at various levels, does not change remarkably the isotopic ratios, unless the maximum temperature $T_{\mathrm{P}}$ is increased.

The subsequent occurrence of extra-mixing on the AGB (followed here through the slow mixing scheme of Model B, as already done in Paper I) adds its effects to those accumulated in the previous RGB stages, and interacts with the abundance changes induced in advanced stages by the third dredge-up. For carbon, the effects of TDU and CBP are opposite, and combine in a complex way. TDU adds fresh ${ }^{12} \mathrm{C}$ from the He shell, while CBP burns it through $p$-captures. The result is a stepwise trend, as shown in Figures 2 and 3. The two figures also illustrate the sensitivity of isotopic ratios to the parameters of the mixing model. Indeed, varying $\dot{M}$ (Figure 2 ) has effects 


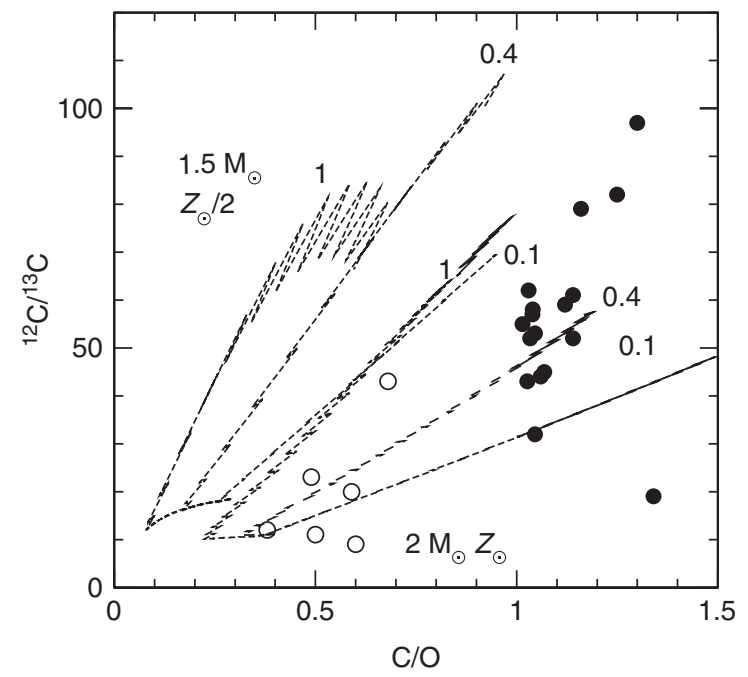

Figure 4 A comparison of model predictions with observations for the carbon isotope and $\mathrm{C} / \mathrm{O}$ ratios, in red giants and AGB stars. The sample of stars is the same for which, in Paper I, with the same mixing scheme and the same set of models, we reproduced the Li abundances. Observations are from Smith \& Lambert (1990); Lambert et al. (1986). The lines refer to the models of a $1.5 \mathrm{M}_{\odot}$ star, with $Z=Z_{\odot} / 3$ (the set of curves extending at higher ${ }^{12} \mathrm{C} /{ }^{13} \mathrm{C}$ ratios) and of a $2-\mathrm{M}_{\odot}$ star with $Z=Z_{\odot}$. Different values of $\dot{M}$ are indicated as labels, in units of $10^{-6} \mathrm{M}_{\odot} \mathrm{yr}^{-1}$. The continuous portions of the curves indicate the phases where the model envelopes are carbon-rich. Observational data are either from M, MS, S giants (open circles) or from $\mathrm{C}(\mathrm{N})$ stars (filled circles).

on $\mathrm{N}, \mathrm{O}$ and $\mathrm{C}$ isotopes, but induces no change on $\mathrm{Ne}$ and Al. On the contrary, changing the maximum temperature $T_{\mathrm{P}}$ (Figure 3 ) mainly affects the $\mathrm{Al}$ isotopic ratio: the representative curves drop below the bottom axis of the graph in the cases with the lower values of $T_{\mathrm{P}}$. A more limited dependence on $T_{\mathrm{P}}$ is shown also by the nitrogen and oxygen isotopic ratios, while the ${ }^{12} \mathrm{C} /{ }^{13} \mathrm{C}$ ratio is seen to vary with $T_{\mathrm{P}}$ only for the case ( $\left.\Delta \log T=0.2\right)$ that samples the minimum of the ${ }^{12} \mathrm{C}$ abundance in the radiative layers.

Figures 4 and 5 show some comparisons with observational data on $\mathrm{CNO}$ abundances. The data points are from Lambert et al. (1986) and from Smith \& Lambert (1990); they refer to stars for which our models were already shown in Paper I to account for the abundance of Li. As is clear from the plot, the curves that refer to the MS-S, O-rich, stages and to the $\mathrm{C}(\mathrm{N}), \mathrm{C}$-rich, ones pass correctly through the area of the observed data. The curves have however a complex behaviour. Figure 4 shows that, before TDU starts, pure extra-mixing above the H-burning shell generates sequences that proceed leftward, reducing the $\mathrm{C} / \mathrm{O}$ and the carbon isotope ratio. When TDU begins to operate this tendency is overcompensated by the addition, at each mixing event, of rather large quantities of ${ }^{12} \mathrm{C}$. These are subsequently partly burned by CBP in the interpulse periods. The interplay of two opposite phenomena is seen also in Figure 5 (showing only models that achieve $\mathrm{C} / \mathrm{O}$ ratios in excess of unity, because the observed points are from C-stars; the case $M=1.5 \mathrm{M}_{\odot}$, $Z=Z_{\odot} / 2$ is not shown, as it never reaches the C-star stage). At each dredge-up ${ }^{12} \mathrm{C}$ increases (transported from

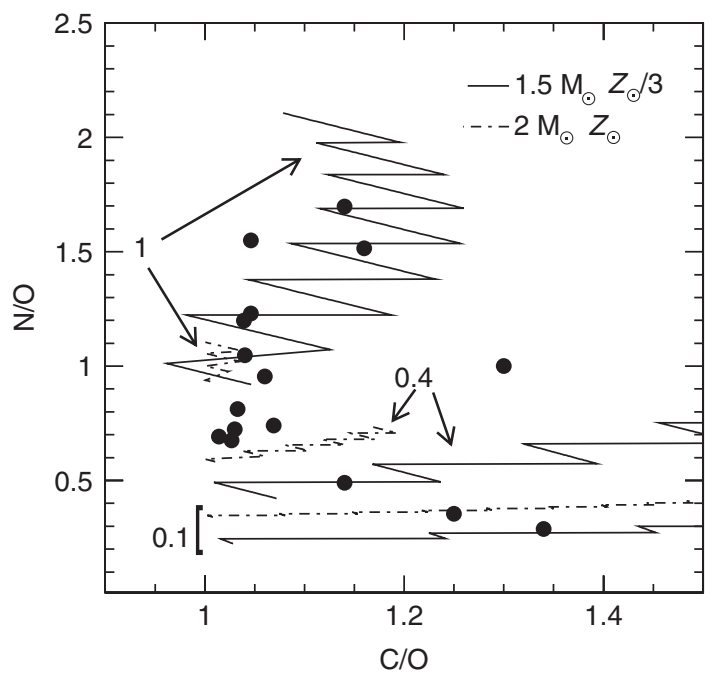

Figure 5 A comparison of model predictions with observations for the N/O and C/O ratios in carbon stars. The sample of $\mathrm{C}$ stars is the same for which in Paper I, with the same mixing scheme and the same set of models, we reproduced the $\mathrm{Li}$ abundances. Observations are from Lambert et al. (1986). The lines refer to the models of a $1.5-\mathrm{M}_{\odot}$ star, with $Z=Z_{\odot} / 3$ (continuous tracks) and of a $2-\mathrm{M}_{\odot}$ star with $Z=Z_{\odot}$ (dash-dotted tracks). Only the parts of the model sequences corresponding to $\mathrm{C} / \mathrm{O}$ ratios larger than unity are shown. Different values of $\dot{M}$ are indicated as labels, in units of $10^{-6} \mathrm{M}_{\odot} \mathrm{yr}^{-1}$.

the He-rich layers) and also a small increase in $\mathrm{N}$ occurs (transported from the inner $\mathrm{H}$-shell layers, where $\mathrm{N}$ is very abundant). In the subsequent interpulse stage CBP burns part of the added carbon, further increasing $\mathrm{N}$. The high $\mathrm{N}$ abundances observed in many $\mathrm{MS}, \mathrm{S}$ and $\mathrm{C}(\mathrm{N})$ giants are produced in this way; the parent stars show only moderate Li enhancements, and this certifies that they are of low mass. Indeed, the alternative mechanism for $\mathrm{N}$ production, hot bottom burning in more massive stars, would efficiently burn carbon (probably avoiding the formation of a $\mathrm{C}$ star) and would produce high $\mathrm{Li}$ abundances, up to $\log \epsilon(\mathrm{Li})=4-5$.

\section{CNO Isotopes in Grains}

Model results need also to be compared with the extensive set of experimental data coming from presolar grains of AGB origin. Examples of such comparisons are presented here as a preliminary check. They are a very small part of what one wants to discuss, but for a question of space we must limit ourselves to a minimum.

A first important constraint to the models comes from Oxide presolar grains, as shown in Figure 6. The Figure shows the evolution followed by the oxygen isotopic mix in the RGB and AGB atmosphere. Similarly to what was obtained by Nollett, Busso \& Wasserburg (2003) with parameterized calculations, also our new models appear to be well suited to explain the composition of oxide grains that depart from the canonical RGB-AGB sequence (represented by the data points and by the dashed line in the upper part of the plot). The lines representing our CBP models descend from the canonical one and reach down to very low ${ }^{18} \mathrm{O} /{ }^{16} \mathrm{O}$ ratios, covering the area occupied by 


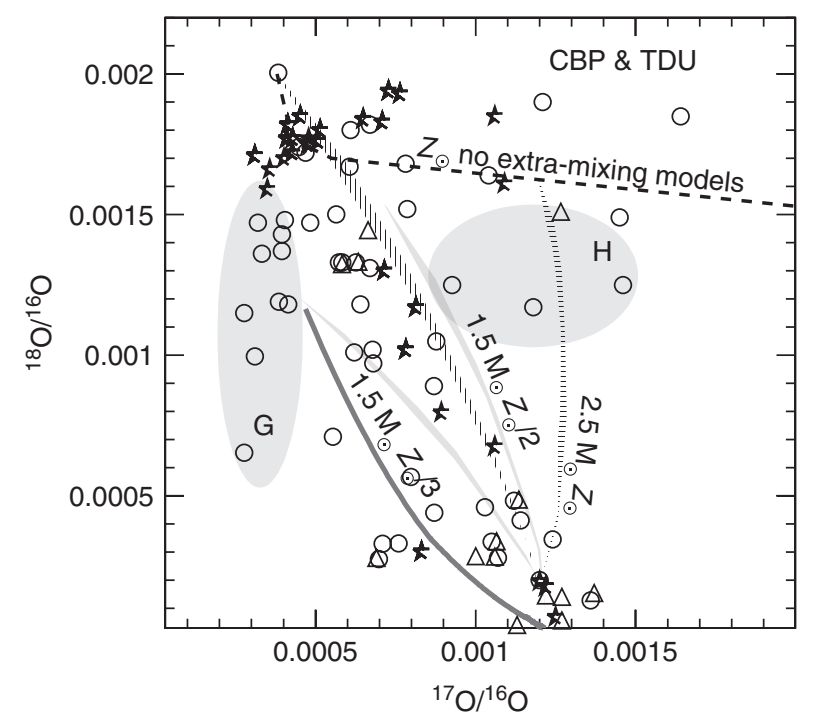

Figure 6 The curves represent the model oxygen isotopic ratios in the envelopes of population I AGB stars, experiencing extra-mixing during the MS-S stage. The dots refer to presolar oxide grains from the quoted references. The sequence labeled 'no extra-mixing' refers to the normal evolution of red giants as described by models without CBP (the higher the stellar mass, the longer the sequence extends toward the right). The other curves refer to our models for different masses and metallicities. Shading along the curves shows the effect of moderate dispersions in the values of $\dot{M}$. Very high $\dot{M}$ values, much higher than considered in Paper I, would bend the curves toward the left. This is shown by the dashed line, where $\dot{M}=10^{-5} \mathrm{M}_{\odot} \mathrm{yr}^{-1}$. We include also a special model, run for a 2.5-M $\odot$ star experiencing the second dredge-up, with the aim of showing the maximum area that CBP models can cover.

presolar grain measurements. Data in the $\mathrm{G}$ area can only be fitted by models of low metallicity, while those in the $\mathrm{H}$ area are from more massive AGB stars, experiencing the second dredge-up and possibly hot bottom burning (we show the track of a representative model doing the second dredge-up for illustrative purposes).

The conclusions are rather obvious: i) population I AGB models can account for the experimental data only if CBP is included; and ii) in order to explain the grains with the lowest content of ${ }^{18} \mathrm{O}$ one needs to perform extensive extra-mixing. If this occurs at high $\dot{M}$ values, then the curvature of model lines bends to the left, as illustrated by the dash-dotted line, run with $\dot{M}=10^{-5} \mathrm{M}_{\odot} \mathrm{yr}^{-1}$.

Figure 7 shows instead a comparison with measurements done in $\mathrm{SiC}$ grains of AGB origin. The relevant models are those reaching $\mathrm{C}$-rich stages in the atmospheres. As some of our models yielding $\mathrm{C} / \mathrm{O}>1$ are of lower-than-solar metallicity, we plot, for comparison, the data not only for 'mainstream' SiC grains (which are believed to come from solar-metallicity stars), but also for the $\mathrm{Y}$ and $\mathrm{Z} \mathrm{SiC}$ grains, thought of as coming from $\mathrm{C}$-rich envelopes of slightly metal-poor AGB stars.

It is clear that, while for the carbon isotopic ratio solar metallicity models well reproduce the mainstream grains, and lower metallicity models occupy the correct region of $\mathrm{Y}$ and $\mathrm{Z}$ grains, the measured nitrogen ratios are at odd with models by a large amount. The discrepancy is at least

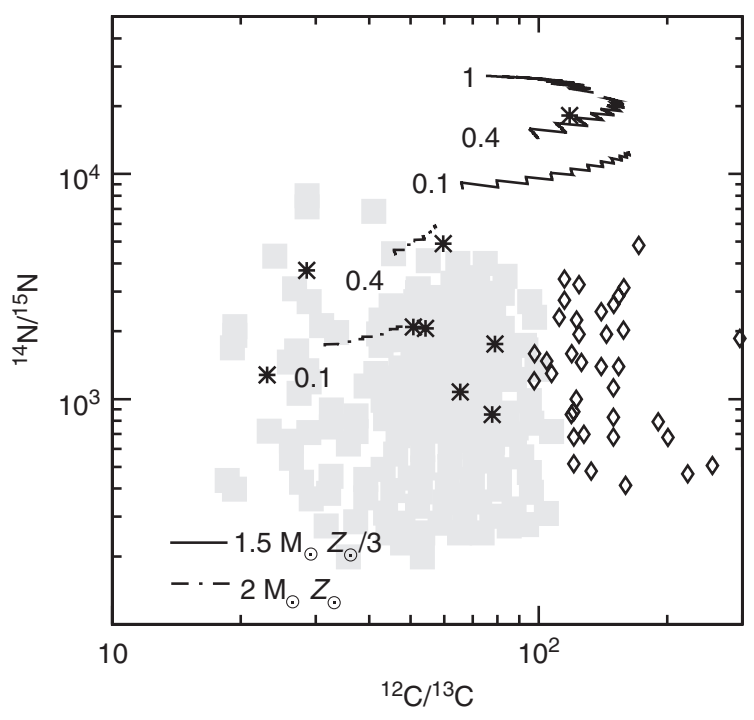

Figure 7 The curves represent model sequences of ${ }^{14} \mathrm{~N} /{ }^{15} \mathrm{~N}$ versus ${ }^{12} \mathrm{C} /{ }^{13} \mathrm{C}$ ratios in the envelopes of population I C-rich AGB stars, experiencing extra-mixing. The $\dot{M}$ values (in units of $10^{-6} \mathrm{M}_{\odot} \mathrm{yr}^{-1}$ ) are shown as labels. The dots and the grey zone refer to presolar $\mathrm{SiC}$ grains from lower-than-solar and solar metallicity stars, respectively (Nittler et al. 1997; Zinner et al. 2007). Only after a large downward shift, of at least a factor of 10 , can the models reproduce the data.

by a factor of ten. We can notice that direct comparisons of the models with nitrogen abundances in stars (Figure 5) suggest that there is no conflict between stellar observations and models (although for stars we do not have the isotopic composition). The impossibility of fitting the lower values of the ${ }^{14} \mathrm{~N} /{ }^{15} \mathrm{~N}$ ratios in $\mathrm{SiC}$ grains is a well known problem (Nollett, Busso \& Wasserburg 2003). The most probable explanation is that the grains undergo a contamination with solar ${ }^{15} \mathrm{~N}$, possibly implanted by the fast winds of the early Sun (J. Duprat, private communication).

In none of the results for CNO nuclei, presented in this or in the previous Section, through the application of our Model B and through comparisons with either stellar observations or presolar grain measurements, the physical origin of the mixing and the velocity of transport play any relevant role. We adopted here the same models used in Paper I for explaining Li abundances, and we can say that they are also suitable for understanding the abundances of CNO nuclei in the same environments; however, only from the need of producing Li we do derive a requirement on the mixing velocity and hence an indication that magnetic buoyancy, allowing for the fast upward transport of H-burning ashes, might be at play, at least at the L-bump on the RGB. Our preliminary study then suggests that coordinated observations of $\mathrm{Li}$ and $\mathrm{CNO}$ isotopes in the same stars are required to constrain the available physical models for extra-mixing, disentangling their different effects.

\section{Conclusions}

In this paper we presented computations of the temporal evolution of abundances at the surface of red giants and AGB stars (especially for $\mathrm{CNO}$ and intermediatemass nuclei), as due to the occurrence of extra-mixing 
processes. We assumed two mixing schemes, applied in sequence: one very fast, occurring at and after the L-bump of the RGB, the second much slower and occurring subsequently. This scenario was suggested by recent works on magnetically-induced mixing in stars presenting a dynamo mechanism (Busso et al. 2007; Denissenkov et al. 2009) and is shown, in an accompanying paper, to yield an explanation for the Li production and destruction in RGB and AGB stars. The scope of our study was to derive the consequences on other nuclei of the extra-mixing history inferred from the $\mathrm{Li}$ constraint and to ascertain whether they agree with the existing record of observations. The results of our (still preliminary) exercise can be summarized as follows:

- It is in general possible to reproduce the $\mathrm{Li}$ and $\mathrm{CNO}$ abundances observed in evolved red giants with models of nucleosynthesis and extra-mixing occurring in the parent stars.

- While explaining CNO isotopes alone does not yield special constraints on the mixing speed (but only on its effectiveness in terms of the transport rate $\dot{M}$ and of the maximum temperature achieved, $T_{\mathrm{P}}$ ), including $\mathrm{Li}$ in the constraints informs us about the velocity with which the processed matter must travel through the radiative layers below the convective envelope. This is so because for producing Li we need to save the parent nucleus ${ }^{7} \mathrm{Be}$ to the envelope before it can undergo $p$ or $e$ captures along the path.

- The joint reproduction of $\mathrm{Li}$ and CNO isotope abundances requires that extra-mixing be driven by a physical process capable of producing both fast and slow transport. The most common diffusive mechanisms, including thermohaline mixing, are not suitable for this task, while the circulation of magnetized matter driven by magnetic buoyancy can do the job.

- Population I AGB stars of low mass are confirmed to be the site where many presolar oxygen-rich and carbonrich grains form. The explanation of the isotopic mix of CNO elements displayed by the grains is a natural consequence of the mixing scheme presented here.

- The above finding has an exception in the $\mathrm{N}$ isotopes of $\mathrm{SiC}$ presolar grains of AGB origin. Their excess in ${ }^{15} \mathrm{~N}$ cannot be reconciled with scenarios based on nucleosynthesis and mixing in low mass stars, and must come from a different source, possibly the injection of ${ }^{15} \mathrm{~N}$ in presolar material during the development of fast winds from the protosun.

- The initial metallicity of the star has direct effects on internal mixing, through the efficiency of TDU, which increases for decreasing metal content. A more indirect effect occurs on CBP: it derives from the change of the abundances induced by TDU, over which proton captures occur in the interpulse phases.

\section{Acknowledgments}

We are indebted to the Italian Ministry of Research for a PRIN grant (n.2006/022731) and to the National Institute of Nuclear Physics (Section of Perugia, ERNA experiment) for providing support and computing facilities.

\section{References}

Amari, S., Zinner, E. \& Lewis, R. S., 2000, M\&PS, 35, 997

Andrews, A. D. et al., 1988, A\&A, 204, 177

Angulo, C. et al., 1999, NuPhA, 656, 3

Boothroyd, A. I., Sackmann, I.-J. \& Wasserburg, G. J., 1995, ApJ, 442, L21

Briand, C. \& Solanki, R. K., A\&A, 330, 1160

Busso, M., Gallino, R. \& Wasserburg, G. J., 1999, ARA\&A 37, 239

Busso, M., Gallino, R., Lambert, D. L., Travaglio, C. \& Smith, C. C., 2001, ApJ, 557, 802

Busso, M., Wasserburg, G. J., Nollett, K. M. \& Calandra, A., 2007, ApJ, 671, 802

Cameron, A. G. W. \& Fowler, W. A., 1971, ApJ, 164, 111

Charbonnel, C., 1994, A\&A, 282, 811

Charbonnel, C. \& Do Nascimento, J. D., Jr, 1998, A\&A, 336, 915

Charbonnel, C. \& Balachandran, S. C., 2000, A\&A, 359, 563

Charbonnel, C. \& Zahn, J.-P., 2007, A\&A, 467, L15

Chin, Y.-N., Henkel, C., Langer, N. \& Mauersberger, R., 1999, ApJ, 512, L143

Choi, B.-G., Huss, G. R., Wasserburg, G. J. \& Gallino, R., 1998, Sci, 282, 1284

Denissenkov, P. A., Pinsonneault, M. \& MacGregor, K. B., 2009, ApJ, in press

Eggleton, P. P., Dearborn, D. S. P. \& Lattanzio, J. C., 2006, Sci, 314, 1580

Gallino, R., Arlandini, C., Busso, M., Lugaro, M., Travaglio, C., Straniero, O., Chieffi, A. \& Limongi, M., 1998, ApJ, 497, 388

Gilroy, K. K., 1989, ApJ, 347, 835

Gilroy, K. K. \& Brown, J. A., 1991, ApJ, 371, 578

Guandalini, R., Palmerini, S., Busso, M. \& Uttenthaler, S., 2009, PASA, this volume

Harris, M. J., Lambert, D. L. \& Smith, V. V., 1988, ApJ, 325, 768

Käppeler, F., Beer, H. \& Wisshak, F., 1989, RPrPh, 52, 945

Lambert, D. L., Gustafsson, B., Eriksson, K. \& Hinkle, K. H., 1986, ApJS, 62, 373

Li, L. H., Sofia, S. \& Belvedere, G., 2005, ApJ, 629, 1164

Meléndez, J. L. \& Ramirez, I., 2007, ApJ, 669, L89

Nittler, L. R., Alexander, C. M. O’D., Gao, X., Walker, R. M. \& Zinner, E., 1997, NuPhA, 621, 113

Nollett, K. M., Busso, M. \& Wasserburg, G. J., 2003, ApJ, 582, 1036

Nordhaus, J., Busso, M., Wasserburg, G. J., Blackman, E. G. \& Palmerini, S., 2008, ApJ, 684, L29

Palacios, A., Charbonnel, C., Talon, S. \& Siess, L., 2006, A\&A, 453, 261

Palmerini, S. \& Busso, M., 2008, NewAR, 52, 412

Sackmann, I.-J. \& Boothroyd, A. I., 1999, ApJ, 510, 217

Shimizu, T. et al., 2008, ApJ, 680, 1467

Smith, V. V. \& Lambert, D. L., 1990, ApJS, 72, 387

Straniero, O., Chieffi, A., Limongi, M., Busso, M., Gallino, R. \& Arlandini, C., 1997, ApJ, 478, 332

Straniero, O., Domínguez, I., Cristallo, S. \& Gallino, R., 2003, PASA, 20, 389

Straniero, O., Gallino, R. \& Cristallo, S., 2006, NuPhA, 777, 311

Švanda, M., Klavn̆a, M. \& Sobotka, M., 2006, A\&A, 458, 301

Švanda, M. et al., 2009, NewA, 14, 429

Wallerstein, G. \& Morell, O., 1994, A\&A, 281, L37

Wasserburg, G. J., Busso, M., Gallino, R. \& Nollett, K. M., 2006, NuPhA, 777, 5

Wasserburg, G. J., Boothroyd, A. I. \& Sackmann, I.-J., 1995, ApJ, 447, L37

Zinner, E. et al., 2007, GeCoA, 71, 4786 